\title{
Diagnostic Emergency CT Imaging Utilization in Our University Hospital Radiology Unit for Pediatrics
}

\author{
Betül Tiryaki Baştuğ* \\ Department of Radiology, Eskişehir Osmangazi University, Turkey
}

*Corresponding author: Betül Tiryaki Baştuğ, Faculty of Medicine, Department of Radiology, Eskişehir Osmangazi University, Eskisehir, Turkey.

\section{Introduction}

Purpose: This study aimed to make the distribution of Computed Tomography (CT) imaging according to organ systems in pediatric emergent patients that admitted to our University Hospital Emergency Radiology Unit.

Methods and Materials: This was a retrospective study comparing resource application between March 2016 and September 2017 in a single ED in a university hospital.

Results: In 32 patient thorax and abdomen CT has studied together for multiple trauma. In 106 head CT was taken for traumatic pathologies. In 34 abdominal CT has studied alone for abdominal emergencies like appendicitis, renal calculi, etc. In 9 orbital CT, in 4 vertebral column CT, in 2 temporal CT, in 13 extremities CT and in 110 head and cervical CT together were done for trauma. In 6 patient head CT with contrast administration was done for suspicion of an intracranial mass. In 10 thorax CT have studied for parenchymal infiltrations whom clinic and laboratory tests were positive. In 1 femoral artery CT angiography was done for detection of traumatic injury.

Conclusion: When the reasons for referral to emergency radiology CT unit of pediatric patients were examined, the most common causes in our center were trauma and abdomen problems.

\section{Introduction}

In many countries there has been an extending use of diagnostic imaging, especially in the emergency departments (ED) [1]. The emergency departments have come to be one of the places where an advanced number of radiologic methods for diagnosing are put on. Ultrasonography (US), Röntgen, Computed Tomography (CT), Magnetic Resonance Imaging (MRI) are the applied radiologic imaging modalities in the assessment of urgent patients. Particularly the expanding ratios of computed tomography (CT) handling in ED seem to vary than in other medical services. Causes of imaging usage are multifactorial. In the present study, we retrospectively reviewed CT imaging volumes in emergency radiology for pediatrics at our institution between March 2016 and September 2017.

\section{Materials and Methods}

This was a retrospective study comparing resource application between March 2016 and September 2017 in a single ED in a university hospital. The study was performed at the Eskişehir Osmangazi University Medical Faculty Hospital which has an academic ED with an annual volume of approximately 70,000 visits. This is a large hospital which is a Level 3 trauma center. Medical information were achieved from the archive in the emergency radiology unit. In this center, emergency radiology service was accessible and open 24 hours a day, seven days a week. Imaging was done after confirmation by a radiologist. The electronic medical archive was examined using a fully automated process. Result 
measures included ED and hospital resource usage, demographics, and clinical characteristics. Radiologic procedures ordered during patients' ED stays were noted ED-based imaging. Demographics (age, sex) and clinical characteristics were gathered. The ED physicians can request exams with radiologist suggestion based on the situation from a set of valid exam protocols. In the analysis of the data, descriptive statistics are presented with frequency, percentage, mean, standard deviation, and minimum and maximum values. Analyzes were made with SPSS 22.0 package program. The study protocol was performed according to the Helsinki declaration.

\section{Results}

Table 1: Patient characteristics that applied CT.

\begin{tabular}{|c|c|c|}
\hline Group & $\mathbf{n}$ & \% \\
\hline Adult & 34353 & 98,5 \\
\hline Pediatric & 509 & 1,5 \\
\hline
\end{tabular}

Table 2: Distribution of CTs according to region.

\begin{tabular}{|c|c|c|}
\hline Group & $\mathbf{n}$ & \% \\
\hline Abdomen & 66 & 12.9 \\
\hline Brain & 252 & 49.5 \\
\hline Vertebra & 134 & 26.3 \\
\hline Thorax & 32 & 6.2 \\
\hline Angiography & 1 & 0.19 \\
\hline Extremity & 13 & 2.5 \\
\hline Orbita & 9 & 1.7 \\
\hline Maxillofacial & 2 & 0.3 \\
\hline
\end{tabular}

Table 3: Gender vs CT regions.

\begin{tabular}{|c|c|c|}
\hline \multirow{2}{*}{ CT Region } & \multicolumn{2}{|c|}{ Gender } \\
\cline { 2 - 3 } & Male & $37,30 \%$ \\
\hline Abdomen & $62,70 \%$ & $0 \%$ \\
\hline Angiography & $100 \%$ & $38,50 \%$ \\
\hline Brain & $61,50 \%$ & $40,70 \%$ \\
\hline Extremity & $59,30 \%$ & $62,10 \%$ \\
\hline Maxillofacial & $37,90 \%$ & $59,90 \%$ \\
\hline Orbita & $40,10 \%$ & $36,20 \%$ \\
\hline Thorax & $63,80 \%$ & $37,90 \%$ \\
\hline Vertebra & $62,10 \%$ & \\
\hline
\end{tabular}

In 32 patient thorax and abdomen CT has studied together for multiple trauma. In 106 head CT was taken for traumatic pathologies. In 34 abdominal CT has studied alone for abdominal emergencies like appendicitis, renal calculi, etc. In 9 orbital CT, in 4 vertebral column CT, in 2 temporal CT, in 13 extremities CT and in 110 head and cervical CT together were done for trauma. In 6 patient head CT with contrast administration was done for suspicion of an intracranial mass. In 10 thorax CT have studied for parenchymal infiltrations whom clinic and laboratory tests were positive. In 1 femoral artery CT angiography was done for detection of traumatic injury (Table 1-3).

\section{Discussion}

There have been huge technological advances in the past decade, not least in diagnostic radiology, with the advent of multidetector CT (MDCT), US, MRI, digital radiography and picture archiving and communication systems (PACS) [2]. The role of the radiologist in trauma and emergency medicine has also dramatically changed [3].

The new generation of MDCT scanners have over 256 detectors, rotation times of less than $0.3 \mathrm{~s}, 0.4 \mathrm{~mm}$ resolution and they can do whole body scans in less than $10 \mathrm{~s}$ and acquiring isotropic voxels [4]. The continuingly evolving software packages process the continuously changing cross sections as the table (gantry) moves through the X-ray circle producing multiplanar reconstructions to allow viewing in any plane with similar resolution and 3D images at the touch of abutton (almost realtime). As the number of applications for CT expand with these newer generation MDCT scanners, so the radiologist's role becomes central in the management of patients, not least in ER. Radiologists are involved not only in interpretation, but also in developing and implementing new protocols that take advantage of the new advances in CT technology. The increase in use has led to an increase in exposure to ionizing radiation and although CT only represents about $20 \%$ of the general workload 
in radiology, it is responsible for over $90 \%$ of the radiation. Recent advances in dose reduction mean that whole body CT scans can now be done with a dose of under $5 \mathrm{mSv}$. Despite this, it is essential that everyone adopts the ALARA (as low as reasonably achievable) principle when using CT and restricts the use in children [5]. MDCT is accurate and cost effective for the evaluation of acute abdominal pain and provides an earlier diagnosis. When surgical intervention may be required, CT provides additional information to facilitate and limit it to the minimum necessary, reducing patient hospital stay and peri-operative morbidity.

\section{Limitations}

Firstly, this was a retrospective cohort study and did not evaluate the decision to perform emergency CT prospectively. Limitations are inherent to retrospective studies based on patient data automatically queried. Data were abstracted using an automated query, and, therefore, it is possible that there were a small number of patients inadvertently not included in the study sample.

\section{Conclusion}

When the reasons for referral to emergency radiology CT unit of pediatric patients were examined, the most common causes in our center were trauma and abdomen problems.

\section{Funding}

None required

\section{Acknowledgement}

None.

\section{Conflict of Interest}

The authors report no conflicts of interest in this work.

\section{References}

1. Mills AM, Raja AS, Marin JR (2015) Optimizing diagnostic imaging in the emergency department. Acad Emerg Med 22(5): 625-631.

2. Rego J, Tan K (2006) Advances in imaging-the changing environment for the imaging specialist. Perm J 10(1): 26-28.

3. Artigas Martín JM, Martí de Gracia M, Claraco Vega LM, Parrilla Herranz $P$ (2015) Radiology and imaging techniques in severe trauma. Med Intensiva 39(1): 49-59.

4. Mori Shinichiro, Endo Masahiro, Tsunoo Takanori, Kandatsu Susumu, Tanada Shuji, et al. (2004) Physical performance evaluation of a 256-slice CT-scanner for four-dimensional imaging. Med Phys 31(6): 1348-1356.

5. Yeung Andy Wai Kan (2019) The "As Low As Reasonably Achievable" (ALARA) principle: a brief historical overview and a bibliometric analysis of the most cited publications. Radioprotection 54(2): 103-109. 